\title{
Planetary Nebulae as Probes of Stellar Populations
}

\author{
Robin Ciardullo \\ Department of Astronomy \& Astrophysics, Penn State University, University Park, PA 16802, \\ USA \\ email:rbc@astro.psu.edu
}

\begin{abstract}
Planetary nebulae $(\mathrm{PNe})$ have the potential to revolutionize our understanding of extragalactic stellar populations. Indeed, in many systems, bright $\mathrm{PNe}$ are the only individual objects identifiable from the ground, and, even more often, they are the only stars that are amenable to spectroscopy. We review the ways in which ensembles of $\mathrm{PNe}$ can be used to probe the metallicity, age, and history of a stellar population. In particular, we discuss three cases: the weak line spectroscopic regime, where one has knowledge of the line-strengths of faint forbidden lines such as [O III] $\lambda 4363$, a bright-line regime, where only the strongest emission lines are visible, and the photometric regime, where the only information available is the [O III] $\lambda 5007$ luminosity function. We show that each of these cases, when properly calibrated, can provide unique insights into the objects that make up a stellar population.
\end{abstract}

Keywords. Planetary nebulae: general, galaxies: stellar content, galaxies: evolution, blue stragglers

\section{Introduction}

In order to understand how galaxies are put together, we must be able to characterize the collective properties of their stars. Information about a system's age (or history of star formation), metallicity (or history of chemical enrichment), initial mass function, and binarity are all critical for modeling the processes which define galactic evolution. Unfortunately, due to the great distances involved, obtaining such data is often extremely difficult.

This is where planetary nebula $(\mathrm{PN})$ research can help. All stellar populations older than $\sim 10^{8} \mathrm{yr}$ make planetary nebulae, and, in a large galaxy, hundreds of PNe are available for study. More importantly, PNe are bright. In systems outside the Local Group, PNe are often the only individual stars that can be identified from the ground, and are almost always the only (non exploding) stars that can be studied spectroscopically. Thus, planetary nebulae can provide unique insights into the history of a stellar system.

The amount of information provided by a population of PNe depends on the type of data that one can acquire. In nearby systems, it is possible to use 4-m and 8-m class telescopes to observe all the important emission lines produced by a $\mathrm{PN}$, including the critical diagnostic lines of [O III] $\lambda 4363$, [S II] $\lambda \lambda 6716,6731$, and [N II] $\lambda 6548,6584$. With this, one can directly measure a population's abundance distribution function and constrain its history of chemical enrichment. In more distant galaxies, these weak emission features are usually unavailable, but one can still use brighter lines such as [O III $] \lambda 5007$, [Ne III] $\lambda 3869, \mathrm{H} \alpha$, and $\mathrm{H} \beta$ to probe stellar content. Finally, if PN spectroscopy is impossible, one can still investigate galactic history via the $[\mathrm{O}$ III] $\lambda 5007$ planetary nebula luminosity function (PNLF). We discuss each of these regimes below. 


\section{The Weak Line Regime}

A decade ago, measurements of the critically important [O III] $\lambda 4363$ emission line were mostly restricted to the PNe of the Galaxy and the Magellanic Clouds. For example, it was only through heroic efforts that Richer, Stasińska, \& McCall (1999) and Jacoby \& Ciardullo (1999) were able to detect this feature in a sizeable sample (42) of PNe in M31's bulge, and thereby measure the population's oxygen abundance. These authors obtained the interesting result that the metallicity spread for M31's bulge stars is over a dex, and that the region's mean oxygen abundance is slightly less than solar. Unfortunately, given the limitations of the 4-m apertures that were available at the time, follow-up studies proved to be impossible.

Today in this era of 8-m class telescopes, it is possible to obtain abundances for hundreds of PNe throughout the largest of the Local Group galaxies, M31 and M33. With these data, one can characterize the systems' abundance distribution functions, not only for oxygen, but for the $\alpha$-process elements of neon, sulfur and argon, all of which are unaffected by stellar evolution. Moreover, because one knows the distances to these extragalactic PNe, the combination of [O III] $\lambda 5007$ photometry and spectrophotometry can be used to derive the objects' absolute line fluxes. This is important, because from this information, it is possible to deduce the locations of the central stars on the HR diagram, estimate their masses (via comparisons with post-AGB evolutionary tracks; Vassiliadis \& Wood 1994), and infer the masses of the stars' progenitors (via the initial mass-final mass relation; Weidemann 2000). In other words, deep spectrophotometry of a large number of planetary nebulae should allow us to trace the chemical enrichment of a stellar population back through time.

Although such an analysis is exciting, it is also extremely difficult. For example, consider the PNe of the Large Magellanic Cloud. Excellent spectrophotometry and nebular models exist for $\sim 60$ objects from the work of Dopita et al. (1991a,b; 1997). If one uses these data to derive $\alpha$-element abundances, and central star luminosities, temperatures, and (ultimately) masses, then a clear trend emerges: PNe from lower-mass (older) progenitors are systematically more metal-poor than their younger counterparts. This suggests that we can, indeed, use PNe to probe a galaxy's history of chemical enrichment. However, if one instead uses ultraviolet measurements of the nebular and stellar continuum to place the central stars on the HR diagram (Herald \& Bianchi 2004; Villaver et al. 2003; Stasińska et al. 2004), then much of this correlation goes away. Clearly, in order to exploit PNe to their fullest, our inferences about central star properties need to be made more robust.

\section{The Bright-Line Regime}

Once outside the Local Group, the [O III] $\lambda 4363$ emission line of planetary nebulae becomes extremely hard, if not impossible, to detect. Although this makes traditional nebular modeling impossible, it does not preclude the use of PNe for population studies. Indeed, Dopita, Jacoby, \& Vassiliadis (1992) and Méndez et al. (2005) have shown that the ratio of [O III] $\lambda 5007$ to $\mathrm{H} \beta$ can be used to place useful constraints on a PN's oxygen abundance.

But one can do better than that. Due to the poor distances of Galactic PNe, samples of Milky Way planetaries are extremely heterogeneous in nature. With high and low-core mass PNe mixed together, it is nearly impossible to separate out planetaries at their peak luminosity from PNe that have already faded by several magnitudes. In extragalactic systems, this is not a problem. Since the absolute luminosities of all the PNe are known, 

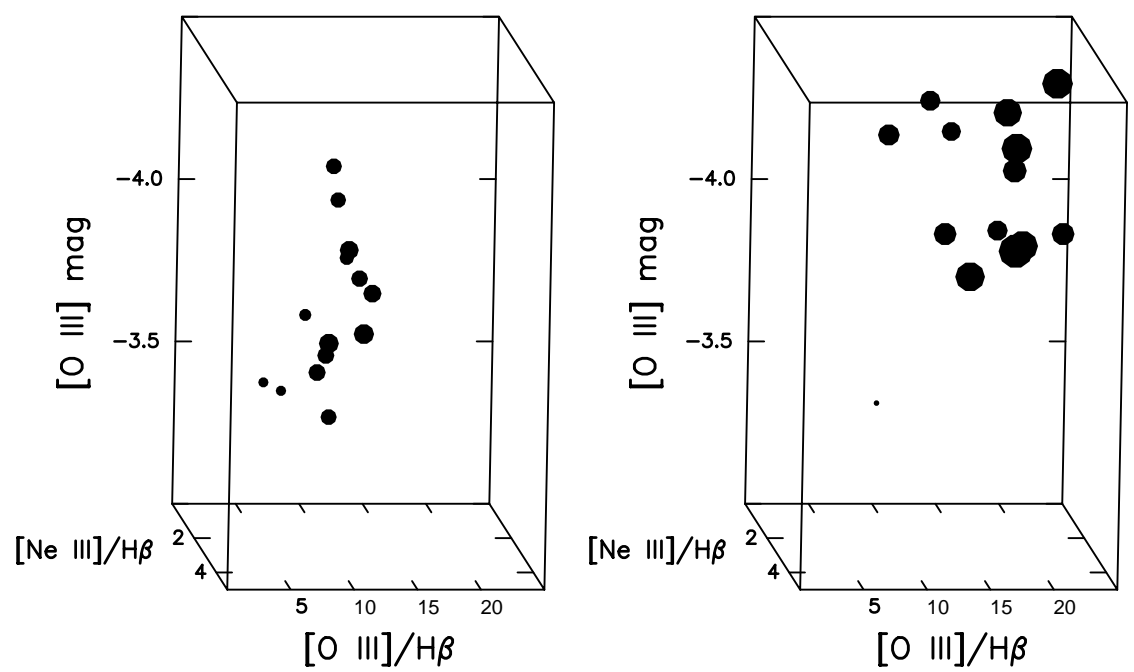

Figure 1. The PNe of the LMC (left) and the large elliptical NGC 4697 (right) plotted in a three-dimensional phase space, with the absolute [O III] magnitudes displayed on the $y$-axis. Note how this type of plot clearly distinguishes the different PN populations.

it is possible to define statistically complete samples of objects at the bright end of the luminosity function and be assured that all are in a similar phase of evolution. For example, PNe in the Milky Way exhibit a wide range of excitation, with [O III] $\lambda 5007$ to $\mathrm{H} \alpha$ emission line ratios $(R)$ ranging from $\sim 4$ to $\sim 0.1$. However, when one studies extragalactic PNe, a pattern emerges: the brightest PNe all have $R>2$, regardless of their parent stellar population (Ciardullo et al. 2002).

In fact, the best way to analyze the bright emission lines from a homogeneous PN dataset is through a differential, multi-dimensional analysis. A schematic example of this approach is shown in Figure 1. In the figure, PNe of the LMC (from Meatheringham \& Dopita 1991a,b) and NGC 4697 (Méndez et al. 2005) are plotted in a 3-dimensional space that has the absolute [O III $] \lambda 5007$ line flux on the $y$-axis, and the $[\mathrm{O} \mathrm{III}] / \mathrm{H} \beta$, and [Ne III] $\lambda 3869 / \mathrm{H} \beta$ line ratios on the $x$ - and $z$-axes. The differences in the populations are obvious. With a reasonable amount of effort, this type of data could be acquired for a range of stellar populations out to $\sim 10 \mathrm{Mpc}$, and serve as the basis for an investigation of $\mathrm{PN}$ production and evolution as a function of galactic environment.

\section{The Photometric Regime}

Spectroscopy of extragalactic PNe is time consuming, and in many cases impossible. Hence one often must rely on [O III] $\lambda 5007$ (or possibly $\mathrm{H} \alpha$ ) photometry to probe a $\mathrm{PN}$ population. Observations in over 50 galaxies have shown that the bright-end of the [O III] $\lambda 5007$ planetary nebula luminosity function is well-described by the truncated exponential

$$
N(M) \propto e^{0.307 M}\left\{1-e^{3\left(M-M^{*}\right)}\right\}
$$

where

$$
M=-2.5 \log F_{5007}-13.74
$$

and $M^{*}=-4.47$ is the absolute magnitude of the most luminous planetary (Jacoby et al. 1992; Ciardullo et al. 2002). Since $M^{*}$ is an excellent standard candle for all but 


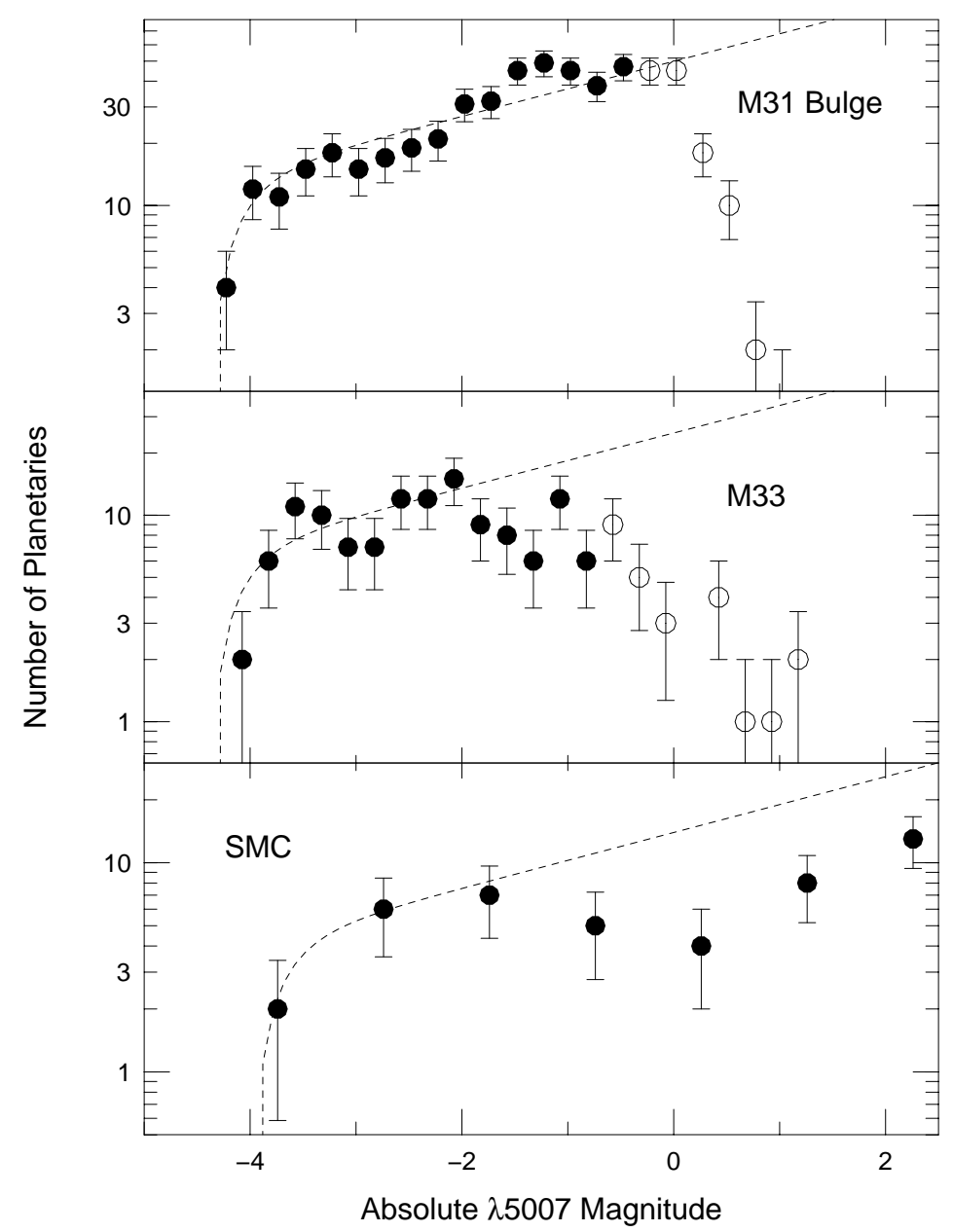

Figure 2. The [O III] $\lambda 5007$ planetary nebula luminosity functions for M31's bulge, M33's disk, and the Small Magellanic Cloud. Note the differences between the populations: star forming systems have a strong deficit of intermediate-brightness planetaries.

the most metal-poor systems, its brightness cannot be used for stellar population studies. However, two properties of the PNLF do hold promise for population measurements.

The first of these is a "dip" in the luminosity function which starts $\sim 2$ mag below $M^{*}$. This feature is not present in every galaxy: in M31, the faint-end of the PNLF is well described by the simple Henize \& Westerlund (1963) exponential, which is expected from an ensemble of uniformly expanding spheres ionized by non-evolving central stars (Ciardullo et al. 1989, 2002). However, as Figure 2 illustrates, star-forming systems such as the Small Magellanic Cloud and M33, have a factor of $\sim 2$ fewer PNe than predicted from the exponential law (Jacoby \& De Marco 2002; Ciardullo et al. 2004). Clearly, this part of the PNLF is sensitive to some aspect of stellar population.

The second population dependent feature of the [O III] PN luminosity function is its normalization. Following Ciardullo et al. (1989) this property is usually parameterized by $\alpha$, the number of PNe detected above a certain absolute magnitude (typically 0.5 or 2.5 mag below $M^{*}$ ), divided by the total (bolometric) luminosity of the stellar parent population. In the star-forming galaxies of the Local Group, $\alpha$ is in good agreement with 
the predictions of stellar evolution models (Buzzoni, Arnaboldi, \& Corradi 2006), but in older stellar populations, this is not always the case. In fact, some elliptical galaxies have almost an order of magnitude fewer PNe than one might expect. This variation has the potential to be an extremely powerful probe of early-type systems.

\section{Explaining the PNLF}

To date, there has been no attempt to simultaneously model both the shape and normalization of the PNLF. Moreover, those models which have concentrated solely on the shape of the luminosity function have failed spectacularly, either by requiring a constant star-formation rate for elliptical galaxies (Méndez \& Soffner 1997) or predicting a bright-end cutoff that is several magnitudes fainter than what is observed (Marigo et al. 2004). Obviously, an important piece of physics is missing from these analyses.

The main difficulty in modeling the PNLF comes from the absolute magnitude of its cutoff. An $M^{*} \mathrm{PNe}$ emits $\sim 600 L_{\odot}$ in its [O III] $\lambda 5007$ emission line. Since no more than $\sim 10 \%$ of the central star's flux can come out in this line, the luminosity of the source powering the nebula must be at least $\sim 6,000 L_{\odot}$. Unfortunately, the only post-AGB cores capable of generating this much energy are those with masses of $M>0.6 M_{\odot}$ (Vassiliadis \& Wood 1994), and these require progenitor stars with $M \gtrsim 2 M_{\odot}$ (Weidemann 2000). Such stars have lifetimes of less than a Gyr, and are not present in any number in elliptical galaxies (e.g., Trager et al. 2005).

As Figure 3 demonstrates, the situation is even worse than it looks. Spectroscopic observations in the Large Magellanic Cloud (Meatheringham \& Dopita 1991a,b) and M31 (Jacoby \& Ciardullo 1999) demonstrate that internal extinction is extremely important in [O III]-bright planetary nebulae. While no $\mathrm{PN}$ is observed to have an [O III] luminosity brighter than $M^{*}$, many PNe have intrinsic [O III] fluxes that are much more luminous than this, sometimes by a factor of $\sim 2$. Such a high luminosity can only be generated by a massive post-AGB core.

In star-forming systems, the presence of high core-mass central stars is not a problem. But what produces these objects in old stellar populations? The number of PNe observed in a galaxy, normalized to the galaxy's underlying (bolometric) luminosity is given by

$$
\alpha=B \cdot \tau \cdot f
$$

where $B$ is the population's luminosity specific stellar evolution flux, $\tau$, the lifetime of the PN stage, and $f$, the fraction of stars which evolve through the PN phase. The value of $B$ is known from the theory of stellar energy generation: all old stellar populations, regard-

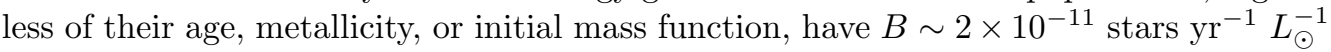
(Renzini \& Buzzoni 1986). The lifetime, $\tau$, is similarly well-constrained, as models show that [O III]-bright PNe spend $\sim 500 \mathrm{yr}$ in the top $0.5 \mathrm{mag}$ of the luminosity function (Marigo et al. 2004). Therefore, observed values of $\alpha$ in the range $\sim 5 \times 10^{-10}<\alpha_{0.5}<$ $3 \times 10^{-9}$ PNe $L_{\odot}^{-1}$ (Ciardullo et al. 2005) translate into stellar fractions of between $5 \%$ and $30 \%$ (see Figure 4). Since absorption line spectroscopy of early-type galaxies place the fraction of young stars far below this value (Trager et al. 2005), the bright PNe of these systems cannot be evolving via normal (single star) stellar evolution. We must look elsewhere for their explanation.

As of this date, two scenarios have been proposed to explain the existence of [O III]bright PNe in early-type galaxies. The first, described by Ciardullo et al. (2005), involves binary coalescence and blue stragglers. Although elliptical galaxies possess few stars with $M \sim 2 M_{\odot}$, they do contain many stars with $M \sim 1 M_{\odot}$. If some of these stars come into contact while still in their hydrogen burning phase, the result may be conservative 


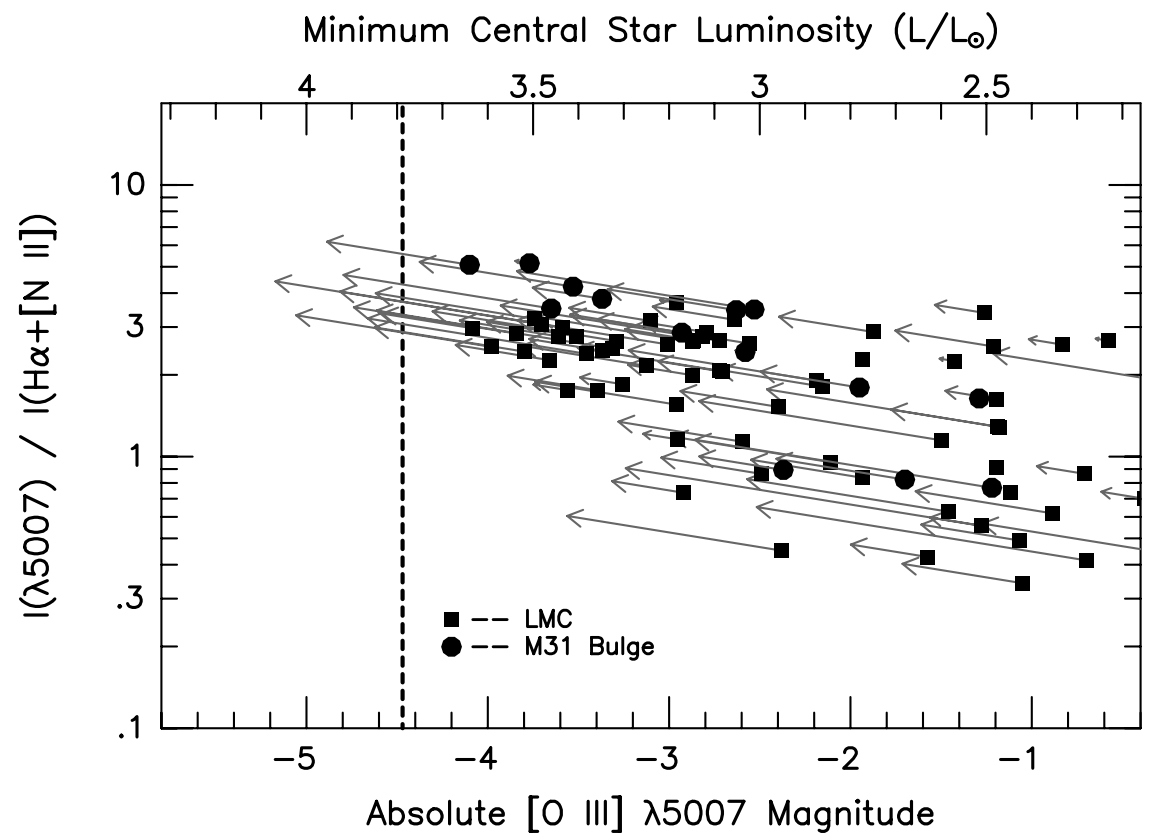

Figure 3. The observed ratio of [O III] $\lambda 5007$ to $\mathrm{H} \alpha$ plotted against [O III] $\lambda 5007$ absolute magnitude for those bright PNe in the LMC and M31 with published spectrophotometry. The arrows display the effects of internal extinction, as estimated from the objects' Balmer decrement. The location of the PNLF cutoff, $M^{*}$, is identified via the dotted line. Note that, although no PN is ever observed to have an [O III] $\lambda 5007$ magnitude brighter than $M^{*}$, many have intrinsic luminosities much brighter than this.

McCrea (1964)-style mass transfer and coalescence. This mechanism, which has been associated with the creation of blue stragglers (Mateo et al. 1990; Carney et al. 2001), has the potential to build the requisite high mass cores, even in systems as old as $\sim 10 \mathrm{Gyr}$.

The blue straggler hypothesis has several advantages. The first, and most obvious, is that such stars are known to exist. In fact, if blue stragglers live $\sim 5 \times 10^{8} \mathrm{yr}$ (Lombardi et al. 2002), then the ratio of [O III]-bright PNe to blue stragglers in systems such as the Ursa Minor dwarf galaxy and the Milky Way halo is approximately the same as that of the objects' lifetimes (Ciardullo et al. 2005). This is consistent with the idea that one object evolves into the other. Moreover, the binary merger hypothesis provides a natural explanation for the observed inverse correlation between $\alpha$ and the excess ultraviolet flux often associated with elliptical galaxies. As a stellar system ages, its main sequence turnoff mass decreases, and this decreases the mass of its post-AGB cores (Weidemann). In the extreme, these low-mass cores will evolve too slowly to form planetary nebulae, and instead produce naked UV-bright sources (Greggio \& Renzini 2000). Meanwhile, the smaller turnoff mass also reduces the number systems capable of merging into stars above the critical $\sim 2 M_{\odot}$ threshold. Consequently the number of $M^{*}$ planetaries will also drop. If this scenario is correct, then $\alpha$ can be used in conjunction with a galaxy's UV upturn to probe the precise location of a population's main-sequence turnoff, even when this turnoff is far below the limit of detectability.

The blue straggler hypothesis only runs into problems when one considers the constraints it imposes on the integrated spectra of elliptical galaxies. As pointed out by Xin \& Deng (2005), a sizeable population of blue stragglers can mimic the presence of young stars, and lead to a significant underestimate for the age of the stellar population. 


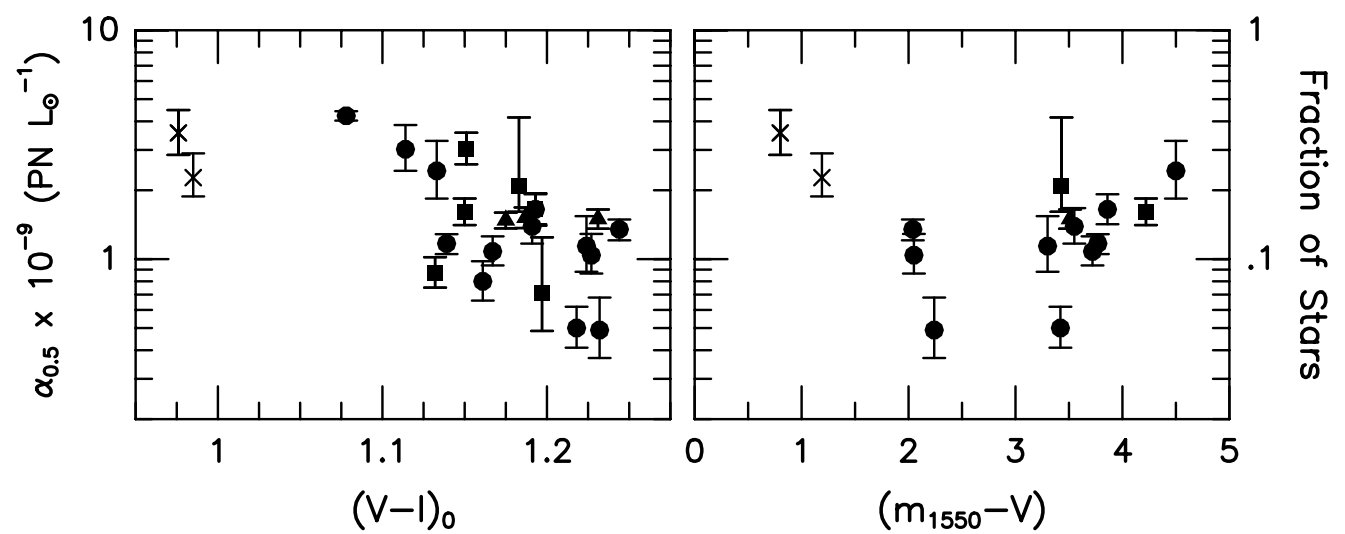

Figure 4. The bolometric-luminosity specific number of PNe in the top 0.5 mag of the [O III] PNLF plotted against the parent galaxy's optical and ultraviolet color. Circles indicate elliptical galaxies, squares are lenticulars, triangles are spiral bulges, and crosses represent systems which contain obvious evidence of star formation. The error bars represent formal $1 \sigma$ uncertainties and are lower limits to the true errors. The right hand axis translates the PN densities to the fraction of stars turning off the main sequence which evolve into [O III]-bright planetaries, under the assumption that the PNe are products of single star evolution.

In fact, Buzzoni, Arnaboldi, \& Corradi (2006) suggest that any blue straggler contribution to an old stellar system must be considerably less than $\sim 10 \%$, in order to avoid measurable effects on the galaxy's colors. However, whether this is a serious problem for models of elliptical galaxies is still an open question.

An alternative scenario for the presence of $M^{*}[\mathrm{O}$ III $] \lambda 5007$ sources in early-type populations involves symbiotic stars. Soker(2006) has proposed that mass transfer from a proto-planetary post-AGB star onto a companion white dwarf can produce the energy and emission lines needed to mimic a bright planetary nebula. This model, which has many commonalities with models of super-soft x-ray sources (albeit with a much higher accretion rate), has the advantage that it does not stress population synthesis predictions: since the lifetime of this accretion phase can be much longer than $\sim 500 \mathrm{yr}$, far fewer [O III] systems are needed to populate the bright end of the luminosity function. However, the model has no natural explanation for the similarity between the maximum brightness of a true planetary nebula and one of these symbiotic sources, nor does it explain the inverse correlation between $\alpha$ and a population's UV upturn.

If there is indeed a second population of objects that evolve through a non-traditional (binary-star) mode of stellar evolution, then the dip in the PNLF has a simple explanation. High core mass planetaries, whether from single star or binary evolution, will always form a luminosity function that has a distinctive dip at intermediate magnitudes. The only way to avoid such a feature is to fill in the dip with lower mass objects. The amplitude of the dip, along with its precise location, would then be a measure of how many of these difficult-to-observe low-mass stars are present in the population and contributing to the PN luminosity function. If this idea is correct, then the agreement between M31's PNLF and the Henize \& Westerlund (1963) exponential law is purely coincidental.

\section{Acknowledgements}

This research made use of the NASA Extragalactic Database and was supported in part by NSF grants AST 00-71238 and AST 03-02030. 


\section{References}

Buzzoni, A., Arnaboldi, M., \& Corradi, R.L.M. 2006, MNRAS, in press

Carney, B.W., Latham, D.W., Laird, J.B., Grant, C.E., \& Morse, J.A. 2001, AJ, 122, 3419

Ciardullo, R., Durrell, P.R., Laychak, M.B., Herrmann, K.A., Moody, K., Jacoby, G.H., \& Feldmeier, J.J. 2004, ApJ, 614, 167

Ciardullo, R., Feldmeier, J.J., Jacoby, G.H., Kuzio de Naray, R., Laychak, M.B., \& Durrell, P.R. 2002, ApJ, 577, 31

Ciardullo, R., Jacoby, G.H., Ford, H.C., \& Neill, J.D. 1989, ApJ, 339, 53

Ciardullo, R., Sigurdsson, S., Feldmeier, J.J., \& Jacoby, G.H. 2005, ApJ, 629, 499

Dopita, M.A., Jacoby, G.H., \& Vassiliadis, E. 1992, ApJ, 389, 27

Dopita, M.A., \& Meatheringham, S.J. 1991a, ApJ, 367, 115

Dopita, M.A., \& Meatheringham, S.J. 1991b, ApJ, 377, 480

Dopita, M.A., Vassiliadis, E., Wood, P.R., Meatheringham, S.J., Harrington, J.P., Bohlin, R.C., Ford, H.C., Stecher, T.P., \& Maran, S.P. 1997, ApJ, 474, 188

Greggio, L., \& Renzini, A. 1990, ApJ, 364, 35

Henize, K.G., \& Westerlund, B.E. 1963, ApJ, 137, 747

Herald, J.E., \& Bianchi, L. 2004, ApJ, 611, 294

Jacoby, G.H., Branch, D., Ciardullo, R., Davies, R.L, Harris, W.E., Pierce, M.J., Pritchet, C.J., Tonry, J.L., \& Welch, D.L. 1992, PASP, 104, 599

Jacoby, G.H., \& Ciardullo, R. 1999, ApJ, 515, 169

Jacoby, G.H., \& De Marco, O. 2002, AJ, 123, 269

Lombardi, J.C., Warren, J.S., Rasio, F.A., Sills, A., \& Warren, A.R. 2002, ApJ, 568, 939

Marigo, P., Girardi, L., Weiss, A., Groenewegen, M.A.T., \& Chiosi, C. 2004, A\& A, 423, 995

Mateo, M., Harris, H.C., Nemec, J., \& Olszewski, E.W. 1990, AJ, 100, 469

McCrea, W.H. 1964, MNRAS, 128, 147

Meatheringham, S.J., \& Dopita, M.A. 1991a, ApJS, 75, 407

Meatheringham, S.J., \& Dopita, M.A. 1991b, ApJS, 76, 1085

Méndez, R.H., Thomas, D., Saglia, R.P., Maraston, C., Kudritzki, R.P., \& Bender, R. 2005, ApJ, 627, 767

Méndez, R.H., \& Soffner, T. 1997, A\&A, 321, 898

Renzini, A., \& Buzzoni, A. 1986, in: ASSL Vol. 122: Spectral Evolution of Galaxies, C. Choisi \& A. Renzini (eds.), (Dordrecht: Reidel), p. 195

Richer, M.G., Stasińska, G., \& McCall, M.L. 1999, A\&A, 135, 203

Soker, N. 2006, ApJ, 640, 966

Stasińska, G., Gräfener, G., Peña, M., Hamann, W.-R., Koesterke, L., \& Szczerba, R. 2004, A\&A, 413, 329

Trager, S.C., Worthey, G., Faber, S.M., \& Dressler, A. 2005, MNRAS, 362, 2

Vassiliadis, E., \& \& Wood, P.R. 1994, ApJS, 92, 125

Villaver, E., Stanghellini, L., \& Shaw, R.A. 2003, ApJ, 597, 298

Weidemann, V. 2000, A\&AA, 363, 647

Xin, Y., \& Deng, L. 2005, ApJ, 619, 824 